\title{
Chapter 5 \\ Damped Oscillatory Behaviors in the Ratios of Stock Market Indices
}

\section{Ming-Chya Wu}

\begin{abstract}
This article reviews a recent finding on the properties of stock market indices (Wu, Europhys Lett 97:48009, 2012). A stock market index is an average of a group of stock prices with equal or unequal weights. Different stock market indices derived from various combinations of stocks are not expected to have fixed relations among them. From analyzing the daily index ratios of Dow Jones Industry Average (DJIA), NASDAQ, and S\&P500 from 1971/02/05 to 2011/06/30 using the empirical mode decomposition, we found that the ratios NASDAQ/DJIA and S\&P500/DJIA, normalized to 1971/02/05, approached and then retained the values of 2 and 1, respectively. The temporal variations of the ratios consist of global trends and oscillatory components including a damped oscillation in 8-year cycle and damping factors of 7183 days (NASDAQ/DJIA) and 138,471 days (S\&P500/DJIA). Anomalies in the ratios, corresponding to significant increases and decreases of indices, are local events appearing only in the time scale less than 8-year cycle. The converge of the dominant damped oscillatory component implies that representative stocks in the pair-markets become more coherent as time evolves.
\end{abstract}

\subsection{Introduction}

The study of financial systems using the concepts and theories developed in physics has attracted much attention in recent years [1-22]. Such study have revealed interesting properties in financial data, which facilitate deeper understanding of the underlying mechanisms of the systems and are essential for sequent modelling. These include financial stylized facts [4, 7-9, 11, 23, 24], such as fat tails in asset return distributions, absence of autocorrelations of asset returns, aggregational normality, asymmetry between rises and falls, volatility clustering [10], phase clustering [13-15], and damped oscillation in ratios of stock market indices [22].

\footnotetext{
M.-C. Wu $(\square)$

Research Center for Adaptive Data Analysis, National Central University, Chungli 32001, Taiwan Institute of Physics, Academia Sinica, Taipei 11529, Taiwan e-mail: mcwu@ncu.edu.tw
}

(C) The Author(s) 2015

H. Takayasu et al. (eds.), Proceedings of the International Conference on Social

Modeling and Simulation, plus Econophysics Colloquium 2014, Springer

Proceedings in Complexity, DOI 10.1007/978-3-319-20591-5_5 
Successful empirical analysis and modelling of financial criticality have suggested possible physical pictures for financial crashes and stock market instabilities $[5,6,12,18-21]$.

In this article, we briefly review our recent study on the damped oscillations in daily stock market indices of Dow Jones Industry Average (DJIA), NASDAQ, and S\&P500, from 1971/02/05 to 2011/06/30 [22]. The daily data were downloaded from Yahoo Finance (http://finance.yahoo.com/), and were preprocessed to have the three indices aligned with the same length by removing three data points in DJIA and S\&P500 (1973/9/26, 1974/10/7, and 1975/10/16) which do not exist in NASDAQ. There are finally 10,197 data points involved in the study. Figure 5.1a shows the daily index data of the three stock markets. It is interesting that by keeping DJIA as a reference and multiplying the NASDAQ index by a factor of 5.2 and S\&P500 by 8.5 , the curves of the rescaled indices coincide very well in several epoches, except large deviations in NASDAQ for the periods 1999-2001 and 2009-2011, as shown in Fig. 5.1b. In year 2011, DJIA is the average price of 30 companies (http://www.djaverages.com/), NASDAQ consists of 1197 companies (http://www.nasdaq.com/), and S\&P500 index is an average result of 500 companies (http://www.standardandpoors.com). Some companies, such as Intel and Microsoft, are included in all the three markets, but most of their compositions are different. The relations among the indices are not crucially determined by the common companies. The coincidence of the three indices via scaling is apparently not trivial, but may result from some kine of coherence among respective representative stocks in the markets. The properties of the relations among them deserve further study.

\subsection{Data Analysis and Discussions}

Let us first consider two indices $x_{i}$ and $x_{j}$. The ratio between them $R_{i j}\left(t_{n}\right)=$ $x_{i}\left(t_{n}\right) / x_{j}\left(t_{n}\right)$ at time $t_{n}$ can be alternatively formulated as

$$
R_{i j}\left(t_{n}\right)=R_{i j}\left(t_{n-1}\right) \frac{1+g_{i}\left(t_{n-1}\right)}{1+g_{j}\left(t_{n-1}\right)},
$$

where

$$
g_{i}\left(t_{n}\right)=\frac{x_{i}\left(t_{n+1}\right)-x_{i}\left(t_{n}\right)}{x_{i}\left(t_{n}\right)},
$$

is the gain of the index $x_{i}$. The gain time series of the three indices are shown in Fig. 5.1c. After normalizing the ratio to the initial value of $R_{i j}$ at $t_{0}$, we have

$$
N R_{i j}\left(t_{n}\right) \equiv \frac{R_{i j}\left(t_{n}\right)}{R_{i j}\left(t_{0}\right)}=\prod_{m=1}^{n} \frac{1+g_{i}\left(t_{m-1}\right)}{1+g_{j}\left(t_{m-1}\right)}
$$


(a)

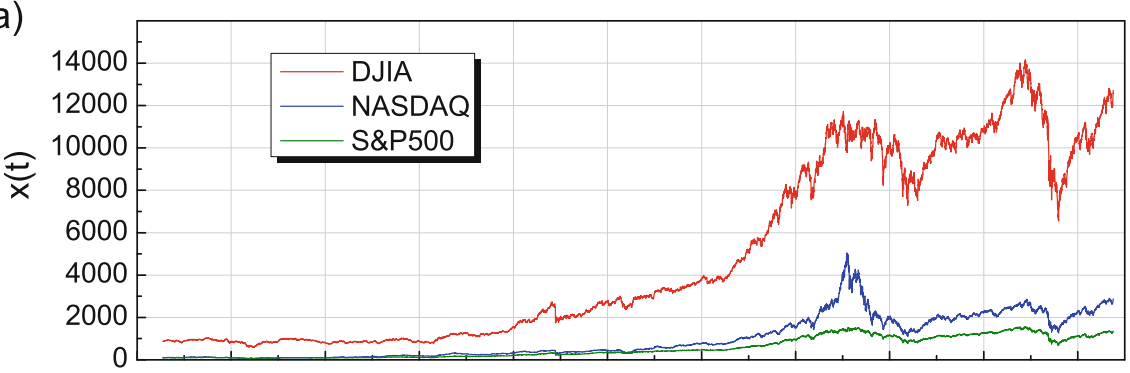

(b)

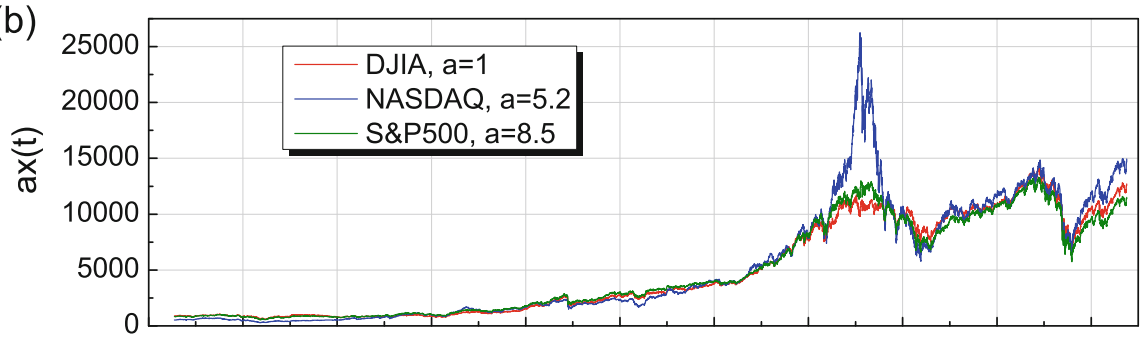

(c)

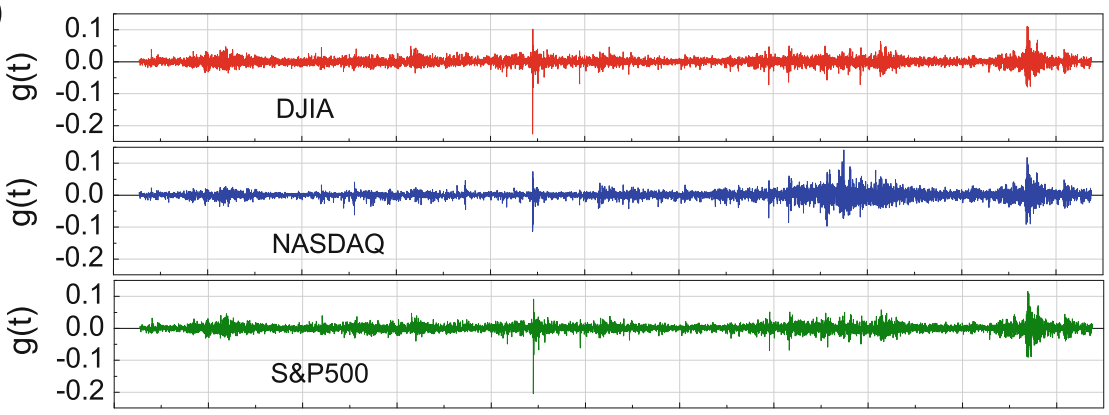

(d)

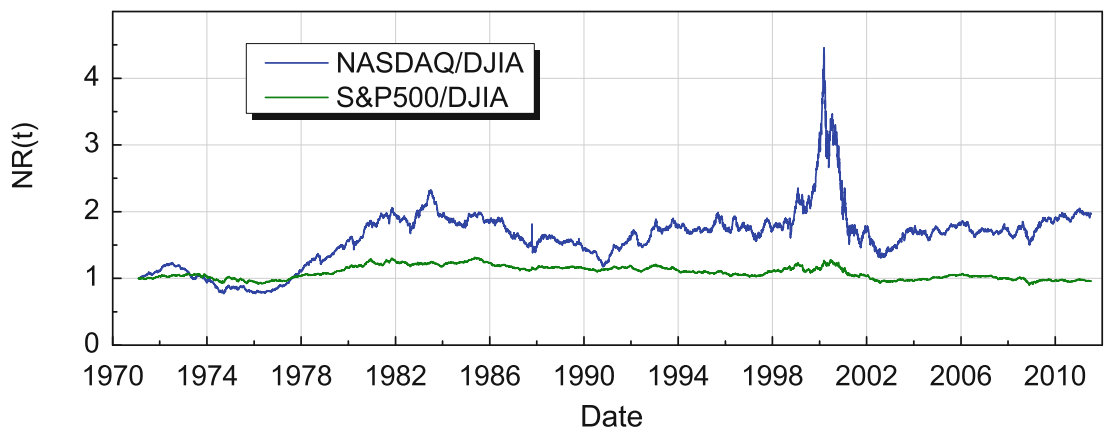

Fig. 5.1 (a) Daily indices of Dow Jones Industry Average (DJIA), NASDAQ, and S\&P500. (b) Rescaled indices $a x(t)$. (c) The gains of the three indices. (d) Ratios of paired indices, normalized to the values on 1971/02/05. (Reproduced from Fig. 1 of [22]) 
Here, $1971 / 02 / 05$ is the initial time for the three indices, and $N R_{i j}\left(t_{n}\right)$ (hereafter abbreviated as $N R(t)$ for simplicity) of NASDAQ/DJIA and S\&P500/DJIA are shown in Fig. 5.1d. The normalized ratio of NASDAQ/DJIA increases before 1982 from 1 to 2 , and then saturated with fluctuations. While there was a sharp peak in 2000, it returned to 1.5 in 2003 and then grew up to 2 gradually. On the other hand, the normalized ratio S\&P500/DJIA varied around 1 with variation magnitudes within \pm 0.3 . Consequently, the general feature of the normalized ratio is that it approached and then retained the values of 2 and 1 for NASDAQ/DJIA and S\&P500/DJIA, respectively. The choice of initial dates for normalization is irrelevant. The scenario is similar to a mechanical system with a "restoring force" acting on it: when the ratio becomes too large or small, it inclines to retain an equilibrium state.

To explore the evolution of the ratios, we analyze the variations of $N R(t)$ in different time scales using the empirical mode decomposition (EMD) [25]. The EMD method assumes that any time series consists of simple intrinsic modes of oscillations [25]. The decomposition explicitly utilizes the actual time series for the construction of the decomposition base rather than decomposing it into a prescribed set of base functions. The decomposition is achieved by iterative "sifting" processes for extracting modes by identification of local extremes and subtraction of local means [25]. The iterations are terminated by a criterion of convergence. Under the procedures of EMD $[25,26]$, the ratio time series $N R(t)$ is decomposed into $n$ intrinsic mode functions (IMFs) $c_{k}$ 's and a residue $r_{n}$,

$$
N R(t)=\sum_{k=1}^{n} c_{k}(t)+r_{n}(t) .
$$

The IMFs are symmetric with respect to the local zero mean and have the same numbers of zero crossings and extremes, or a difference of 1, and all the IMFs are orthogonal to each other [25]. According to the algorithm of EMD, $c_{1}$ is the highest frequency component, $c_{2}$ has a frequency about half of $c_{1}$, and so on. Ideally, the frequency content of each component is not overlapped with others such that the characteristic frequencies of all components are distinct. Thus one component can then be characterized by its own range of periods in time domain. Here, both the $N R$ of NASDAQ/DJIA and S\&P500/DJIA are decomposed into ten components. Using the property that each component has a distinct period, we summed over different components to assess the behaviors of the ratios in different time scales. Among others, IMFs $c_{6}$ to $c_{9}$ are of special interest for their average time scales estimated by zero-crossing calculations are larger than 1 year (about 250 transaction days, which is a suitable time scale to analyze the behaviors in Fig. 5.1d. Figures 5.2a and 5.3a show the comparisons of $N R$, residue $r_{9}$ and combinations of the residue and IMFs, $c_{9}+r_{9}$ and $c_{(6-9)}+r_{9}$ (here $c_{6}+c_{7}+c_{8}+c_{9}$ has been abbreviated as $c_{(6-9)}$ for simplicity). For the current case, we are more interested in the residue $r_{9}$ and IMFs $c_{8}$ and $c_{9}$, shown in Figs. 5.2b and 5.3b. The residue $r_{9}$ is the trend of the ratio NASDAQ/DJIA which approaches 2 gradually from 1.2 (Fig. 5.2b), while $r_{9}$ of 
(a)

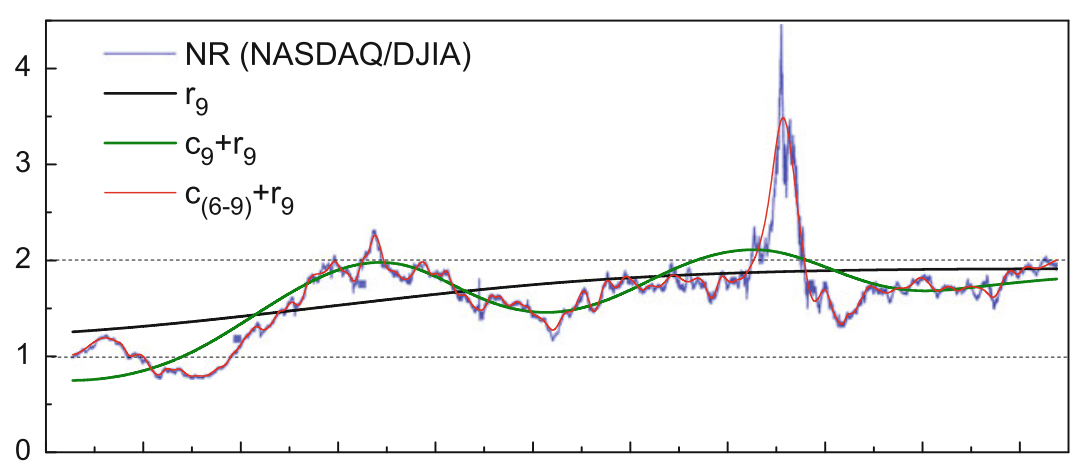

(b)
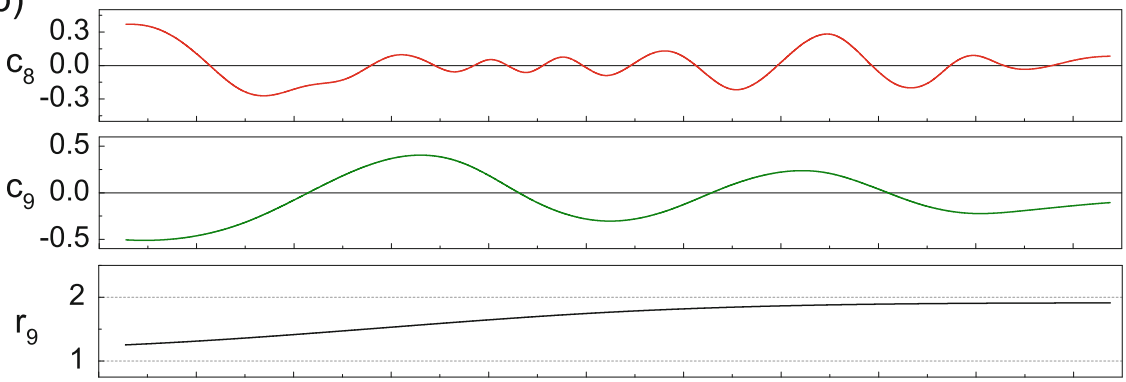

(c)

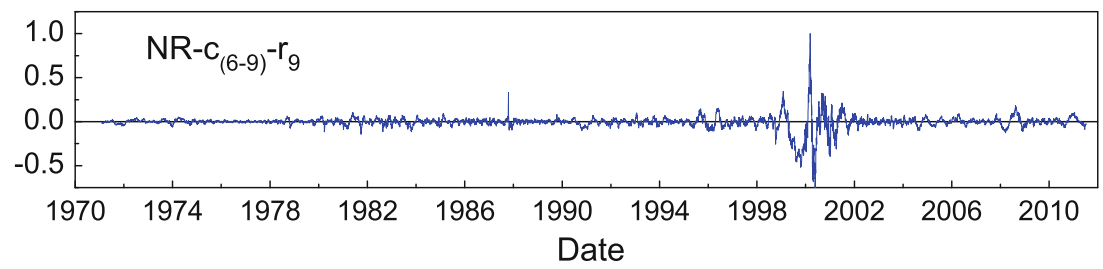

Fig. 5.2 Empirical mode decomposition (EMD) of the ratio $N R$ for NASDAQ/DJIA. (a) Comparisons of $N R$, IMFs and IMF combinations. (b) IMFs $c_{8}, c_{9}$, and residue $r_{9}$. (c) The data of $N R-c_{6}-c_{7}-c_{8}-c_{9}-r_{9}$. (Reproduced from Fig. 2 of [22])

S\&P500/DJIA grows up from 1 to 1.2 and then decreases back to 1 (Fig. 5.3b). With the aid of zero-crossing calculations and fitting, the IMF $c_{9}$ reveals that the variations of the ratios in the scale of 8-year cycle behave as a damped oscillation in the form of $\exp \left[-\left(t_{n}-t_{0}\right) / \gamma\right]$ with damping factors $\gamma \approx 7183$ days (NASDAQ/DJIA) and 138,471 days (S\&P500/DJIA) determined from the local minima of IMF $c_{9}$. Thus, the combination of $c_{9}$ and $r_{9}$ shows the converge of oscillations to values 2 and 1 for NASDAQ/DJIA and S\&P500/DJIA, respectively. Meanwhile, the IMF $c_{8}$ corresponding to (2-4)-year cycle is accompanied with frequency modulation in late of 1990s, implying the trigger of the anomaly in amplitude change and its recovery to regular situation lasts 1.5 oscillatory cycles, about $4-6$ years. Since this anomaly does not appear in IMF $c_{9}$, it is a local event in time with time scale less than 8-year cycle. Here we should remark that the nature of the EMD method is adaptive. It 
(a)

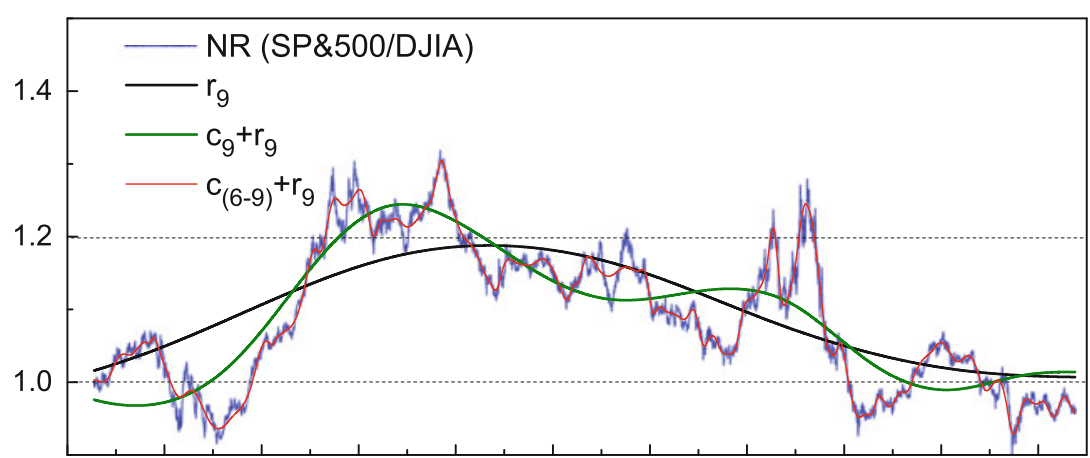

(b)
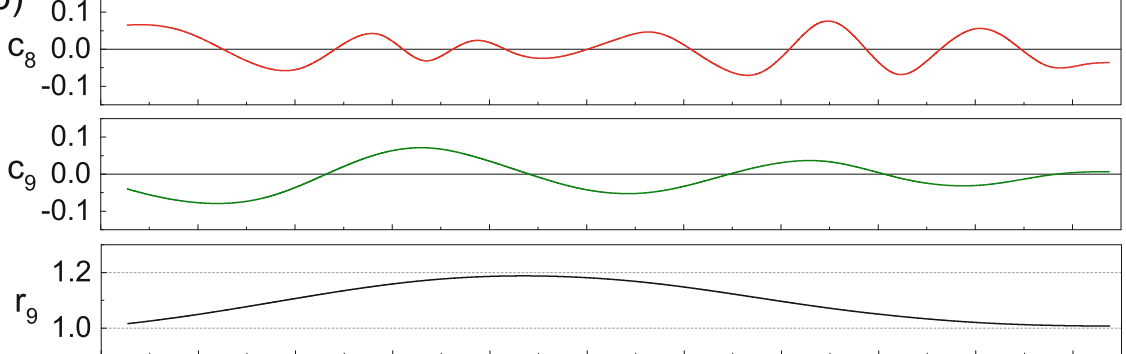

(c)

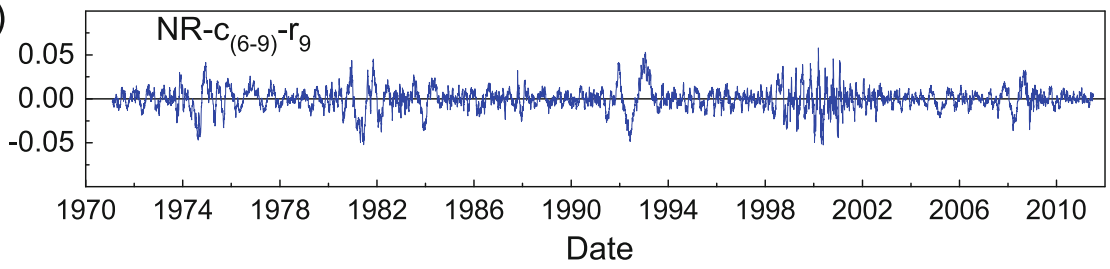

Fig. 5.3 Empirical mode decomposition (EMD) of the ratio $N R$ for S\&P500/DJIA. (a) Comparisons of $N R$, IMFs and IMF combinations. (b) IMFs $c_{8}, c_{9}$, and residue $r_{9}$. (c) The data of $N R-c_{6}-c_{7}-c_{8}-c_{9}-r_{9}$. (Reproduced from Fig. 3 of [22])

catches intrinsic oscillations in a time series, such that the number of IMFs depends on the properties of the data itself (i.e., the index of IMFs may change). The abovediscussed behaviors can be observed no matter the data used here is considered as a whole or is split into two or more segments (if long enough to see components with particular time scales) for the same analysis.

The components of the ratios in the cycle less than half year (about 125 days) are derived by subtracting $c_{(6-9)}+r_{9}$ from $N R$. The data of $\delta N R=N R-c_{(6-9)}-r_{9}$ are shown in Figs. 5.2c and 5.3c for NASDAQ/DJIA and S\&P500, respectively. Within cycles less than 1 year, there are no explicit repeat patterns in the data. Thus, we analyze their statistical properties by the detrended fluctuation analysis (DFA) [2729] and the multiscale entropy (MSE) [30] analysis, and the results are presented in Fig. 5.4. The DFA analysis measures the fluctuation $F(n)$ of $\delta N R$ with respect to a 

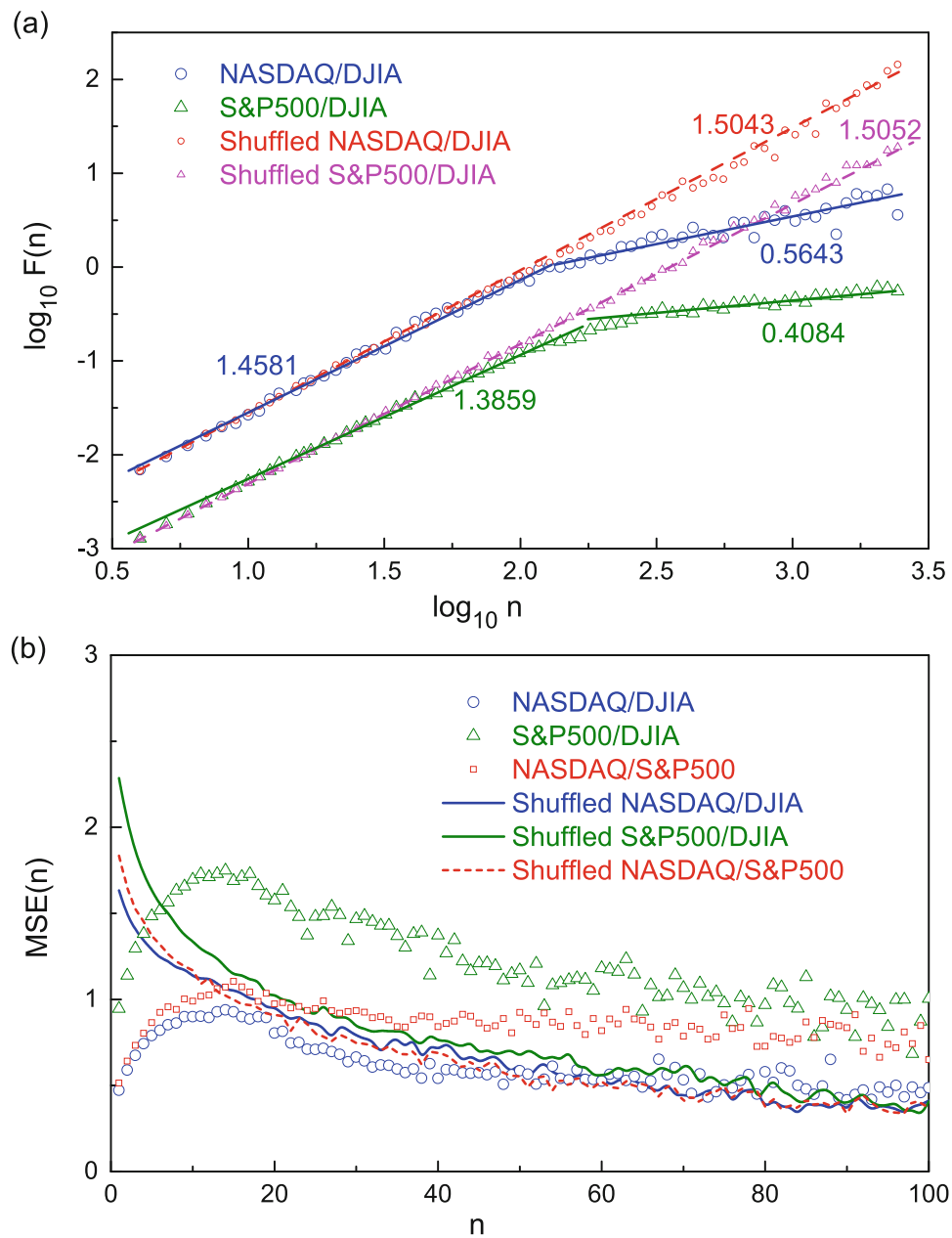

Fig. 5.4 Statistical properties of $N R-c_{(6-9)}-r_{9}$. (a) Detrended fluctuation analysis (DFA). The numbers indicate the $\alpha$ values of the linear segments. (b) Multiscale entropy (MSE) analysis. The shuffled data are generated by randomizing $N R-c_{(6-9)}-r_{9}$ using normal distribution. (Edited from Fig. 4 of [22])

linear fit of the data $\left(\delta N R_{n}\right)$ in a time window $n$, and use an index $\alpha$ defined from

$$
F(n)=\sqrt{\frac{1}{T} \sum_{t}\left[\delta N R(t)-\delta N R_{n}(t)\right]^{2}} \sim n^{\alpha}
$$

to describe the correlation property of the data [27-29]. The results of $\alpha=1.4851$ for NASDAQ/DJIA and $\alpha=1.3859$ for S\&P500/DJIA in Fig. 5.4a suggest that the property of $N R-c_{(6-9)}-r_{9}$ is similar to a Brownian motion with more negative 
correlation $(<1.5)$ in the time scale less than half year (125 days) in Fig. 5.4a, indicating the anti-persistent behaviors in the ratios. For reference, the DFA analysis for the shuffled $N R-c_{(6-9)}-r_{9}$ data of NASDAQ/DJIA and S\&P500/DJIA is also shown in Fig. 5.4a. The shuffled data manifests the property of Brownian motion with $\alpha=1.5$. The relatively stronger anti-persistent behavior in S\&P500/DJIA than in NASDAQ/DJIA is considered as a signature of more significant self-adjustment in the ratio of S\&P500/DJIA. The change of slope at 125 days is due to the removal of high-order IMFs $\left(c_{(6-9)}\right.$ and $\left.r_{9}\right)$. The slopes in this regime indicate that effective changes of the ratios in S\&P500/DJIA $(\alpha=0.4084)$ is smaller than in NASDAQ/DJIA $(\alpha=0.5643)$.

Next, the MSE analysis measures the scale dependence of the complexity in the data [30]. Higher complexity corresponds to a higher information content or a superiority of system control [31]. The analysis is implemented by calculating the entropies of a set of resampled data in different window sizes, which is to be a scale factor in MSE plot, according to

$$
\begin{aligned}
s_{n}(t) & =\sum_{\delta N R_{n}}(t) P\left(\overline{\delta N R_{n}}(t)\right) \log \left[P\left(\delta \overline{N R_{n}}(t)\right)\right], \\
\overline{\delta N R_{n}}(t) & =\frac{1}{n} \sum_{i=1}^{n-1} \delta N R(t+i), 1 \leq i \leq \frac{T}{n},
\end{aligned}
$$

where $P\left(\overline{\delta N R_{n}}(t)\right)$ is the occurrence probability of the value $\overline{\delta N R_{n}}(t)$. MSE is an average of successive difference of $s_{n}(t)$ over time. The analysis is finally presented by the curve of MSE as a function of $n$. Here, the relative complexity of the data is evaluated with respect to a reference defined from the corresponding shuffled data or some standard noises. The results in Fig. 5.4b show that the information content of $N R-c_{(6-9)}-r_{9}$ of NASDAQ/DJIA is richer than that of S\&P500/DJIA in all time scales. Remarkably, both of the MSE curves reach maxima at about 14 days, implying reassessments on ratios are relatively more active in this time scale. The entropy of $N R-c_{(6-9)}-r_{9}$ for NASDAQ/DJIA is lower than the shuffled data, generated by randomizing the time series of $N R-c_{(6-9)}-r_{9}$ using normal distribution, in the scale less than 60 days, and that for S\&P500/DJIA is less than the shuffled data in the scale less than 7 days. Interestingly, the information content in $N R-c_{(6-9)}-r_{9}$ for NASDAQ/DJIA is relatively lower than the corresponding shuffled data resembling to a white noise. There is a weaker correlation between NASDAQ and DJIA than between S\&P500 and DJIA. As a result, larger deviations of the rescaled indices in Fig. 5.1b for DJIA and NASDAQ than DJIA and S\&P500 can be observed in the period from 1999 to 2002. Here for reference, the same analysis applied to $N R-c_{(6-9)}-r_{9}$ of NASDAQ/S\&P500 is also presented in Fig. 5.4b, which shows that the data for NASDAQ/S\&P500 also reaches maximum at about 14 days and is less than its shuffled data in the scale less than 12 days.

We further calculate the dynamical cross-correlations for pairs of the stock market indices using logarithmic return, $l r_{i}\left(t_{n}\right)=\log \left[x_{i}\left(t_{n+1}\right) / x_{i}\left(t_{n}\right)\right]$. The dynamical 
cross-correlation between returns of two indices is defined as

$$
\rho_{i j}\left(t_{n}\right)=\frac{\left(l r_{i}-\left\langle l r_{i}\right\rangle\right)\left(l r_{j}-\left\langle l r_{j}\right\rangle\right)}{\sigma_{i} \sigma_{j}},
$$

with $\sigma_{i}^{2}=\left\langle l r_{i}^{2}-\left\langle l r_{i}\right\rangle^{2}\right\rangle$ the variance of the index, and $\langle\cdots\rangle$ indicates an average over a time window $T$. Despite of phase differences in short time scale, the variations of the indices in large time scale are generally positive correlated (more in phase). Figure 5.5a shows the window size dependence of the average correlation of the stock indices. The average correlation between S\&P500 and DJIA is stronger than NASDAQ and DJIA for all window sizes, consistent with inference from the MSE analysis in Fig. 5.4b that the information content in \&P500/DJIA in the cycle less than half-year is richer than NASDAQ/DJIA. DJIA and NASDAQ have the strongest correlation at $T=60$ days, while the correlation strength between DJIA and S\&P500 grows gradually with time and saturates at $T>1000$ days.

Note that the normalized ratio $N R_{i j}\left(t_{n}\right)$ in Eq. (5.3) can be rewritten as

$$
N R_{i j}\left(t_{n}\right)=\frac{1+\sum_{k=1}^{n} G_{i}^{(k)}}{1+\sum_{k=1}^{n} G_{j}^{(k)}},
$$

with

$$
G_{i}^{(k)}=\frac{1}{k !} \sum_{t_{m_{1}} \neq t_{m_{2}} \ldots} \prod_{l=1}^{k} g_{i}\left(t_{m_{l}}\right) .
$$

The term $G_{i}^{(1)}$ is a sum of all the gains. The means of the grains are 0.00032095 , 0.00040822, and 0.00031729 for DJIA, NASDAQ, and S\&P500, respectively, and the value of $G_{i}^{(1)}$ is in the order of 1 . The term $G_{i}^{(2)}$ is proportional to the autocorrelation function of the gain, defined as $C(\tau)=\int_{t_{0}}^{t_{N}-\tau} \delta g(t) \delta g(t+\tau) d t / \eta^{2}$, with variance $\eta^{2}=\left\langle g^{2}-\langle g\rangle^{2}\right\rangle$, and its value is also in the order of 1 . The $G_{i}^{(k)}$,s with $k \geq 3$ are combinations of the sum of gains and autocorrelation functions. Further calculations of $G_{i}^{(k)}$ show that the values of all $G_{i}^{(k)}$ 's of Eq. (5.10) are in the order of 1. Consequently, all $G_{i}^{(k)}$ 's have equal contributions to the ratios. We then calculate the autocorrelation of the absolute gain and the results are shown in Fig. 5.5b. Using exponential decay model to fit the autocorrelation function, the correlation length is determined to be 194 days for DJIA, 766 days for NASDAQ, and 238 days for S\&P500, which are less than 4 years. Consequently, from above analysis, we confirmed that the damped oscillation in 8-year cycle is not a consequence of crosscorrelation and autocorrelation of the indices. 
(a)

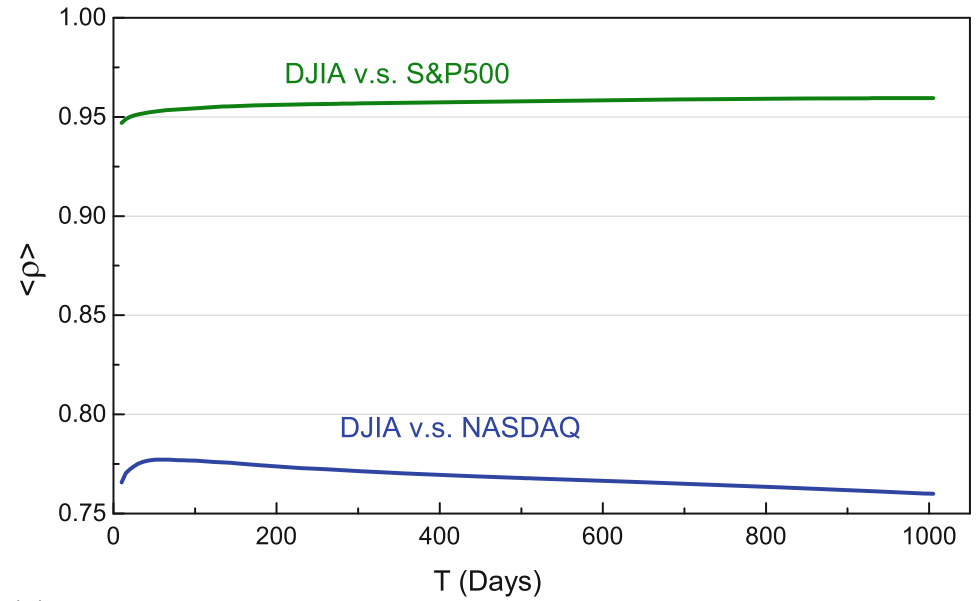

(b)

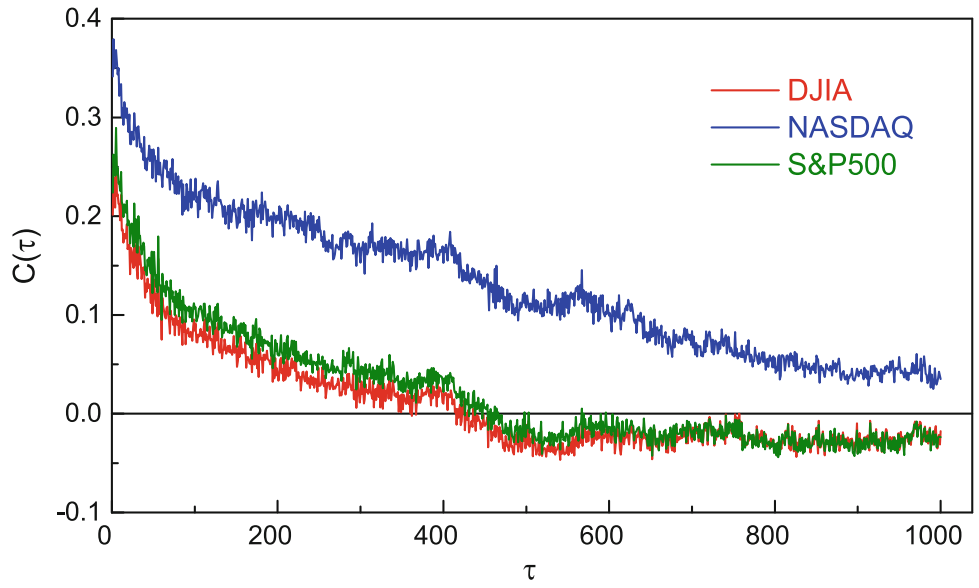

Fig. 5.5 (a) Window size dependence of the average dynamic cross-correlation stock market indices. DJIA and NASDAQ has the strongest correlation at $T=60$ days, and the correlation between DJIA and S\&P500 grows gradually with time and saturates at $T>1000$ days. (Edited from Fig. 5c of [22]) (b) Autocorrelation functions of the absolute gains. The correlation length is 194 days for DJIA, 766 days for NASDAQ, and 238 days for S\&P500. (Edited from Fig. 6b of [22])

\subsection{Conclusion}

In conclusion, from analyzing the ratios of the daily index data of DJIA, NASDAQ, and S\&P500 from 1971/02/05 to 2011/06/30, it can be shown that though three indices are distinct from one another, using suitable scaling factors, the indices can be made coincidence very well in several epoches, except NASDAQ in the periods 1999-2001 and 2009-2011. Sophisticated time series analysis based on 
EMD method further shows that the ratios NASDAQ/DJIA and S\&P500/DJIA, normalized to 1971/02/05, approached and then retained 2 and 1, respectively, from 1971 to 2011, through damped oscillatory components in 8-year cycle and damping factors of about 29 years (7183 days for NASDAQ/DJIA) and 554 years (138,471 days for S\&P500/DJIA). Note that the damped oscillation of 8-year cycle is not associated with the characteristic time scales in the auto-correlation of the gains and cross-correlation of the returns of the indices. Furthermore, the peak of NASDAQ/DJIA in the period from 1998 to 2002, which is considered as an anomaly in the ratio, is a local event that does not appear in the 8-year cycle. The converge of the damped oscillatory component implies that representative stocks in the pairmarkets become more coherent as time evolves. For the components with cycles less than half-year, behaviors of self-adjustments are observed in the ratios, and there is a relatively active reassessment on the ratio in the time scale of 14-days according to the results of MSE analysis. The behavior of self-adjustment in the ratio for S\&P500/DJIA is more significant than in NASDAQ/DJIA.

Finally we would like to remark that the damped components found in the study set reasonable bounds to the variations of the indices. It may be informative for risk evaluation of the markets. This requires further investigations.

Acknowledgements This work was supported by the Ministry of Science and Technology (Taiwan) under Grant No. MOST 103-2112-M-008-008-MY3.

Open Access This book is distributed under the terms of the Creative Commons Attribution Noncommercial License which permits any noncommercial use, distribution, and reproduction in any medium, provided the original author(s) and source are credited.

\section{References}

1. Laloux L, Cizeau P, Bouchaud JP, Potters M (1999) Phys Rev Lett 83:1467

2. Plerou V, Gopikrishnan P, Rosenow B, Amaral LAN, Stanley HE (1999) Phys Rev Lett 83:1471

3. Mantegna RN (1999) Eur Phys J B11:193

4. Mantegna RN, Stanley HE (2000) An introduction to econophysics, correlations and complexity in finance. Cambridge University Press, Cambridge

5. Sornette D, Johansen A (2001) Quant Finan 1:452

6. Sornette D (2003) Phys Rep 378:1

7. Bouchaud JP, Potters M (2003) Theory of financial risk and derivative pricing: from statistical physics to risk management. Cambridge University Press, Cambridge

8. Gabaix X, Gopikrishnan P, Plerou V, Stanley HE (2003) Nature 423:267

9. McCauley JL (2004) Dynamics of markets: econophysics and finance. Cambridge University Press, Cambridge

10. Yamasaki K, Muchnik L, Havlin S, Bunde A, Stanley HE (2005) Proc Natl Acad Sci USA 102:9424

11. Lax M, Cai W, Xu M (2006) Random processes in physics and finance. Oxford University Press, Oxford

12. Kiyono K, Struzik ZR, Yamamoto Y (2006) Phys Rev Lett 96:068701

13. Wu M-C, Huang M-C, Yu H-C, Chiang TC (2006) Phys Rev E 73:016118 
14. Wu M-C (2007) Phys A 375:633

15. Wu M-C (2007) J Korean Phys Soc 50:304

16. Bouchaud JP (2008) Nature 455:1181

17. Podobnik B, Stanley HE (2008) Phys Rev Lett 100:084102

18. Sornette D, Woodard R, Zhou W-X (2009) Phys A 388:1571

19. Preis T, Reith D, Stanley HE (2010) Phil Trans R Soc A 368:5707

20. Preis T (2011) Eur Phys J Special Topics 194:5

21. Preis T, Schneider JJ, Stanley HE (2011) Proc Natl Acad Sci USA 108:674

22. Wu M-C (2012) Europhys Lett 97:48009

23. Cont R (2001) Quant Finan 1:223

24. Engle RF, Patton AJ (2001) Quant Finan 1:237

25. Huang NE, Shen Z, Long SR, Wu MC, Shih HH, Zheng Q, Yen N-C, Tung C-C, Liu HH (1998) Proc R Soc Lond A 454:903

26. Wu M-C, Hu C-K (2006) Phys Rev E 73:051917

27. Peng C-K, Buldyrev SV, Havlin S, Simons M, Stanley HE, Goldberger AL (1994) Phys Rev E 49:1685

28. Chen Z, Ivanov PC, Hu K, Stanley HE (2002) Phys Rev E 65:041107

29. Chen Z, Hu K, Carpena P, Bernaola-Galvan P, Stanley HE, Ivanov PC (2005) Phys Rev E 71:011104

30. Costa M, Goldberger AL, Peng C-K (2002) Phys Rev Lett 89:062102

31. Costa M, Goldberger AL, Peng C-K (2005) Phys Rev E 71:021906 